\title{
Hybrid Analog-Digital Precoding Revisited Under Realistic RF Modeling
}

\author{
Adrian Garcia-Rodriguez, Student Member, IEEE, Vijay Venkateswaran, Member, IEEE, \\ Pawel Rulikowski, Member, IEEE, and Christos Masouros, Senior Member, IEEE
}

\begin{abstract}
In this letter, we revisit hybrid analog-digital precoding systems with emphasis on the modeling of their radiofrequency (RF) losses, to realistically evaluate their benefits in 5G system implementations. We focus on fully-connected analog beamforming networks (FC-ABFNs) and on discrete Fourier transform implementations, and decompose these as a bank of commonly used RF components. We then model their losses based on their S-parameters. Our results reveal that the performance and energy efficiency of hybrid precoding systems are severely affected once these, commonly ignored, losses are considered in the overall design. In this context, we also show that hybrid precoder designs similar to Butler matrices are capable of providing better performances than FC-ABFN for systems with a large number of RF chains.
\end{abstract}

Index Terms-Analog beamforming networks, hybrid precoding, millimeter wave, massive MIMO, Butler matrix.

\section{INTRODUCTION}

5 G COMMUNICATION systems are expected to incorporate a large number of antennas at the base stations (BS) for serving a multiplicity of user terminals while satisfying their data rate requirements [1], [2]. For instance, both millimeter-wave (mmWave) and large-scale antenna systems (LSAS) exploit their large number of antennas for compensating the severe path loss at high frequencies and providing a favourable propagation, respectively [1], [2]. In turn, the substantial increase in the number of antennas has prompted the development of strategies to reduce the hardware and signal processing complexities required by future communications systems.

Hybrid analog-digital precoding and detection schemes aim at reducing the number of RF chains by translating part of the signal processing operations to the analog domain, which is crucial in mmWave systems [2], [3]. In this context, a variety of ABFN have been recently proposed [2]-[4]. However, they generally disregard the practical implications of signal processing in the RF domain such as the additional power losses introduced by the ABFN. These power losses have been partly considered with oversimplifying assumptions in [5] and observed in the measurements of [6], where their impact

Manuscript received May 20, 2016; revised July 13, 2016; accepted August 4, 2016. Date of publication August 10, 2016; date of current version October 11, 2016. This work was supported in part by the Royal Academy of Engineering, U.K., and in part by the EPSRC under Grant EP/M014150/1. The associate editor coordinating the review of this paper and approving it for publication was S. K. Mohammed.

A. Garcia-Rodriguez and P. Rulikowski are with Nokia Bell Labs, Dublin 15, Ireland (e-mail: adrian.garcia_rodriguez@ nokia.com; pawel.rulikowski@nokia.com).

V. Venkateswaran was with Alcatel-Lucent Bell Labs, Dublin 15, Ireland. He is now with Huawei Technologies, Stockholm 164 94, Sweden (e-mail: vijay.venkateswaran@huawei.com).

C. Masouros is with the Department of Electronic and Electrical Engineering, University College London, London, WC1E 7JE, U.K. (e-mail: c.masouros@ucl.ac.uk).

Digital Object Identifier 10.1109/LWC.2016.2598777 on the rates or the energy efficiency has not been analyzed. Instead, in this letter we comprehensively characterize the impact of considering realistic $\mathrm{ABFN}$ in the performance of hybrid precoding schemes. In particular, our contributions can be summarized as follows:

- We model ABFN as a bank of elementary RF components by relying on their S-parameters, which relate to the transfer function of the hybrid precoder RF subsystem [7]. The analysis of FC-ABFN reveals that there are significant power losses even with ideal components.

- Subsequently, we incorporate the insertion losses (IL) found in real analog hardware components for assessing the effective performance of hybrid precoding systems. We show that, contrary to existing intuition, the combination of these losses results in a saturation in the spectral efficiency of the transmission with increasing RF chains.

\section{HYBRID PRECODING UNDER REALISTIC RF MODELING}

Let us consider a BS comprised of $N$ antennas transmitting towards $K \leq N$ single-antenna users. The BS implements $N \geq$ $N_{\mathrm{RF}} \geq K \overline{\mathrm{RF}}$ chains and hybrid analog-digital precoding for transmission. This system can be characterized as

$$
\mathbf{y}=\mathbf{H}^{\mathrm{H}} \mathbf{F} \mathbf{s}+\mathbf{w}=\mathbf{H}^{\mathrm{H}} \mathbf{F}_{\mathrm{RF}} \mathbf{F}_{\mathrm{BB}} \mathbf{s}+\mathbf{w},
$$

where $\mathbf{y} \in \mathbb{C}^{K \times 1}$ represents the received signals, $\mathbf{F} \in \mathbb{C}^{N \times K}$ is the composite precoding matrix, $\mathbf{s} \in \mathbb{C}^{K \times 1} \sim \mathcal{C} \mathcal{N}\left(\mathbf{0}, \frac{1}{K} \mathbf{I}_{K}\right)$ comprises the modulated data symbols and $\mathbf{w} \in \mathbb{C}^{K \times 1} \sim$ $\mathcal{C} \mathcal{X}\left(\mathbf{0}, \sigma^{2} \mathbf{I}_{K}\right)$. Moreover, $\mathbf{h}_{k}^{\mathrm{H}}$ collects the frequency-flat channel gains between the BS antennas and the $k$-th user, where $\mathbf{h}_{k}=\mathbf{R}_{k}^{\frac{1}{2}} \mathbf{z}_{k}$ denotes the $k$-th column of $\mathbf{H} \in \mathbb{C}^{N \times K}$ [4]. Here, $\mathbf{z}_{k} \in \mathbb{C}^{N \times 1} \sim \mathcal{C} \mathcal{N}\left(\mathbf{0}, \mathbf{I}_{N}\right)$ and $\mathbf{R}_{k} \in \mathbb{C}^{N \times N}$ represents the channel covariance matrix. Additionally, $\mathbf{F}_{\mathrm{BB}} \in \mathbb{C}^{N_{\mathrm{RF}} \times K}$ represents the digital baseband precoding matrix and $\mathbf{F}_{\mathrm{RF}} \in \mathbb{C}^{N \times N_{\mathrm{RF}}}$ characterizes the RF ABFN, as represented in Fig. 1. For simplicity, the availability of perfect channel state information (CSI) at the receivers is assumed in the following.

Traditional hybrid system models normalize the composite precoding matrix $\mathbf{F}$ for satisfying a sum power constraint and subsequently apply additional constraints to the RF precoder $\mathbf{F}_{\mathrm{RF}}$. Instead, in this contribution we aim at deriving the constraints of $\mathbf{F}_{\mathrm{RF}}$ based on the specific hardware implementation of the ABFN. Specifically, we solely impose $\left\|\mathbf{F}_{\mathrm{BB}}\right\|_{\mathrm{F}}^{2}=K$ and define $\rho \triangleq \frac{K}{\sigma^{2}}$ as the normalized signal-to-noise ratio (SNR). These constraints guarantee a fair comparison between hybrid and fully digital systems (i.e., when $N_{\mathrm{RF}}=N$ and $\mathbf{F}_{\mathrm{RF}}=\mathbf{I}_{N}$ ), since the constraints to $\mathbf{F}_{\mathrm{RF}}$ will arise naturally from our S-parameter analysis of the ABFN. The signal-to-interference-plus-noise ratio (SINR) of the $k$-th user is given by [8]

$$
\gamma_{k}=\frac{\left|\mathbf{h}_{k}^{\mathrm{H}} \mathbf{f}_{k}\right|^{2}}{\sum_{i \neq k}\left|\mathbf{h}_{k}^{\mathrm{H}} \mathbf{f}_{i}\right|^{2}+\sigma^{2}},
$$

This work is licensed under a Creative Commons Attribution 3.0 License. For more information, see http://creativecommons.org/licenses/by/3.0/ 


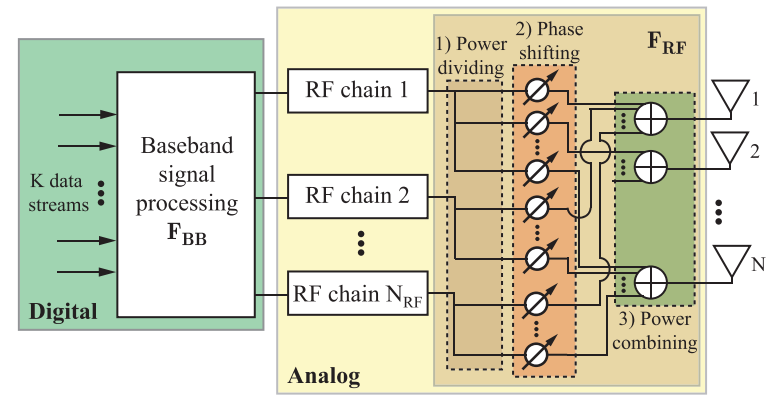

Fig. 1. Block diagram of a hybrid precoding system comprised of digital precoding and a fully-connected analog beamforming network (FC-ABFN).

where $\mathbf{f}_{k} \in \mathbb{C}^{N \times 1}$ represents the $k$-th column of $\mathbf{F}$. The ergodic sum rates in bits per second can be expressed as [8]

$$
R_{\text {sum }}=B S_{\mathrm{e}}=B \sum_{k=1}^{K} \mathbb{E}\left[\log _{2}\left(1+\gamma_{k}\right)\right]
$$

where $B$ denotes the system bandwidth and $S_{\mathrm{e}}$ represents the sum spectral efficiency (SE).

In the following we derive realistic models for characterizing two ABFN architectures: Fully-connected and DFT-based ABFN. These models are based on the holistic electromagnetic design, implementation and measurements of the hybrid architectures developed in [6] for obtaining a realistic comparison between fully digital solutions and practical hybrid precoders.

\section{A. Fully-Connected Analog Beamforming} Networks (FC-ABFN)

The architecture of a FC-ABFN is shown in Fig. 1, where three stages can be clearly identified: a first one comprised of power dividers where each of the $N_{R F}$ input signals is divided into $N$ equal-power outputs characterized by the matrix $\mathbf{F}_{\mathrm{D}} \in \mathbb{C}^{\left(N_{\mathrm{RF}} \cdot N\right) \times N_{\mathrm{RF}}}$, a subsequent one where $N_{\mathrm{RF}} \cdot N$ signals are phase shifted represented by $\mathbf{F}_{\mathrm{PS}} \in \mathbb{C}^{\left(N_{\mathrm{RF}} \cdot N\right) \times\left(N_{\mathrm{RF}} \cdot N\right)}$, and a final stage where $N_{\mathrm{RF}}$ signals are combined with power combiners and coupled to $N$ antenna ports characterized by $\mathbf{F}_{\mathrm{C}} \in$ $\mathbb{C}^{N \times\left(N_{\mathrm{RF}} \cdot N\right)}$. Based on the above, we propose to decompose the analog beamforming matrix for FC-ABFN as [6]

$$
\mathbf{F}_{\mathrm{RF}}=\mathbf{F}_{\mathrm{C}} \cdot \mathbf{F}_{\mathrm{PS}} \cdot \mathbf{F}_{\mathrm{D}} \text {. }
$$

Since the decomposition of the FC-ABFN in (4) is performed in the RF domain, an accurate description of its operation should be based on understanding the RF characteristics of its hardware components. For this reason we define the signal distribution in $\mathbf{F}_{\mathrm{RF}}$ based on the S-parameter representation of the hardware components involved in $\mathbf{F}_{\mathrm{C}}, \mathbf{F}_{\mathrm{PS}}$ and $\mathbf{F}_{\mathrm{D}}$. Specifically, $\mathbf{F}_{\mathrm{D}}$, which is comprised of Wilkinson power dividers [7], can be modeled following a block diagonal structure

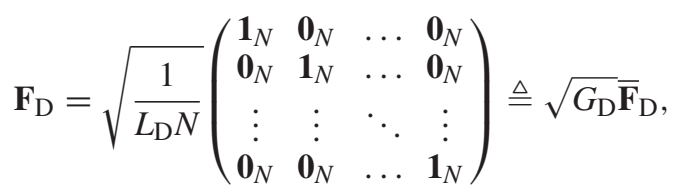

where $L_{\mathrm{D}}$ corresponds to the substrate or static power loss [7], $G_{\mathrm{D}} \triangleq 1 /\left(L_{\mathrm{D}} N\right)$ and $\mathbf{1}_{T} \in \mathbb{N}^{T \times 1}$ and $\mathbf{0}_{T} \in \mathbb{N}^{T \times 1}$ represent the all-ones and all-zeros vectors respectively. Similarly, we characterize the phase shifting network matrix $\mathbf{F}_{\mathrm{PS}}$ as

$$
\mathbf{F}_{\mathrm{PS}}=\sqrt{1 / L_{\mathrm{PS}}} \cdot \operatorname{diag}\left(\left\{f_{1,1}, f_{2,1}, \ldots, f_{N, N_{\mathrm{RF}}}\right\}\right) \triangleq \sqrt{G_{\mathrm{PS}}} \overline{\mathbf{F}}_{\mathrm{PS}},
$$

where $L_{\mathrm{PS}}$ denotes the static power losses introduced by each phase shifter [7], $G_{\mathrm{PS}} \triangleq L_{\mathrm{PS}}^{-1}$ and the coefficient $f_{i, j}, \forall i \in$ $\{1, \ldots, N\}, j \in\left\{1, \ldots, N_{\mathrm{RF}}\right\}$ corresponds to the $i, j$-th phase shift of $\mathbf{F}_{\mathrm{RF}}$ normalized to satisfy $\left\|f_{i, j}\right\|=1$. The specific phase shifting values $\mathbf{F}_{\mathrm{PS}}$ can be obtained following multiple design criteria (see [2]-[4]). Moreover, we express the combining matrix $\mathbf{F}_{\mathrm{C}}$ as

$$
\mathbf{F}_{\mathrm{C}}=\sqrt{\frac{1}{L_{\mathrm{C}} N_{\mathrm{RF}}}}\left[\operatorname{diag}\left(\mathbf{1}_{N}\right), \ldots, \operatorname{diag}\left(\mathbf{1}_{N}\right)\right] \triangleq \sqrt{G_{\mathrm{C}}} \overline{\mathbf{F}}_{\mathrm{C}},
$$

where $G_{C} \triangleq 1 /\left(L_{C} N_{R F}\right)$. Two factors can be observed with respect to the losses in (7): First, $L_{C}$ represents the static power losses introduced by the power combiners [7]. Secondly, the S-parameter representation of passive RF components reveals additional losses in the form of the scaling coefficient $1 / \sqrt{N_{\mathrm{RF}}}$ [7, Ch. 7]. In other words, the adaptive nature of $\mathbf{F}_{\mathrm{BB}}$ and the data symbols produces phase and amplitude mismatches in the signals at the input of the power combiners, hence introducing a loss in the signal combining process due to their non-coherent addition [9] - an aspect not often considered in the related literature. We refer to this mismatch leading to loss among fixed connections in $\mathbf{F}_{\mathrm{RF}}$ as dynamic power loss [6], [7], and we remark that it arises even for ideal analog hardware components. Substituting (5)-(7) into (4) we finally have

$$
\mathbf{F}_{\mathrm{RF}}=\sqrt{G_{\mathrm{C}} G_{\mathrm{PS}} G_{\mathrm{D}}} \overline{\mathbf{F}}_{\mathrm{C}} \overline{\mathbf{F}}_{\mathrm{PS}} \overline{\mathbf{F}}_{\mathrm{D}} \triangleq \sqrt{G_{\mathrm{T}}^{\mathrm{FC}}} \mathbf{F}_{\mathrm{RF}}^{\mathrm{FC}} .
$$

Remark: The consideration of the dynamic power losses entails that, in contrast with fully digital precoding, the power amplifiers will have to compensate for signal-dependent losses in order to guarantee a given transmission power [6]. Indeed, (7) manifest power losses that depend on the amplitude and phase imbalances of the signals at the input of the power combiners [7], [9], and can scale linearly with $N_{\mathrm{RF}}$ in ideal FCABFN. These power losses severely degrade the performance of practical FC-ABFN implementations with large $N_{\mathrm{RF}}$.

\section{B. DFT Analog Beamforming Networks (DFT-ABFN)}

While fully-connected networks allow designing arbitrary $\mathrm{ABFN}$, the above analysis has revealed that power combiners introduce substantial power losses. To alleviate these, we propose to consider 4-port hybrid directional couplers. This is because, in contrast with Wilkinson combiners, these components ideally preserve the power introduced at their input, even when the input signals have different phases and amplitudes [6], [7]. However, while reducing the power losses, designs with hybrid couplers can degrade performance because they have a more restrictive definition of $\mathbf{F}_{\mathrm{RF}}$ as described below, hence posing a trade-off that we aim at studying in this letter. Specifically, 4-port hybrid couplers facilitate implementations of the DFT in the RF domain, commonly referred to as Butler matrices [6], [10]. An illustrative example of a $4 \times 4$ Butler matrix is shown in Fig. 2, where their structure comprised of $N_{\mathrm{HYB}}=\left\lceil\log _{2}(N)\right\rceil$ and $N_{\mathrm{PS}}=\left\lceil\log _{2}(N)\right\rceil-1(N \geq$ 2 ) hybrid coupling and phase shifting stages can be observed. Here, $\lceil\cdot\rceil$ rounds to the largest closest integer. Based on the above, $\mathbf{F}_{\mathrm{RF}}$ can be expressed in the case of DFT-ABFN as

$$
\mathbf{F}_{\mathrm{RF}}=\frac{1}{\sqrt{\left(L_{\mathrm{PS}}\right)^{N_{\mathrm{PS}}}\left(L_{\mathrm{HYB}}\right)^{N_{\mathrm{HYB}}}}} \mathbf{F}_{\mathrm{RF}}^{\mathrm{DFT}} \triangleq \sqrt{G_{\mathrm{T}}^{\mathrm{DFT}}} \mathbf{F}_{\mathrm{RF}}^{\mathrm{DFT}},
$$

where $L_{\mathrm{HYB}}$ are the static power losses introduced by each hybrid power coupler and $\mathbf{F}_{\mathrm{RF}}^{\mathrm{DFT}} \in \mathbb{C}^{N \times N_{\mathrm{RF}}}$ is a submatrix of 


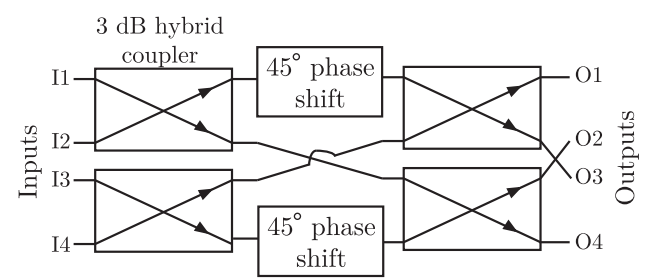

Fig. 2. Block diagram of a $4 \times 4$ Butler matrix.

TABLE I

Orientative INSERTION LOSSES (IL) OF THE HARdWARE COMPONENTS EMPLOYED IN THE DESIGN OF ANALOG BEAMFORMING NETWORKS

\begin{tabular}{|c||c|c|}
\hline- & Millimeter-wave & Sub 5 GHz \\
\hline \hline $\begin{array}{c}\text { Three-port power dividers / } \\
\text { combiners }\left(\bar{L}_{\{\mathrm{D}, \mathrm{C}\}}\right)\end{array}$ & $0.6 \mathrm{~dB}[13]$ & $0.5 \mathrm{~dB}[14]$ \\
\hline Hybrid couplers & $0.5 \mathrm{~dB}[15]$ & $0.15 \mathrm{~dB}[14]$ \\
\hline Phase shifters & $0.5 \mathrm{~dB}[15]$ & $3.5 \mathrm{~dB}[16]$ \\
\hline
\end{tabular}

the $\mathbb{C}^{N \times N}$ DFT matrix [4]. For uniform linear arrays (ULA), $\mathbf{F}_{\mathrm{RF}}^{\mathrm{DFT}}$ can be defined by approximating $\mathbf{R}_{k}$ by a circulant matrix $\mathbf{C}_{k}$ and selecting the eigenvectors corresponding to its largest eigenvalues [4]. We remark that the power loss characterization of (9) has been verified via the RF simulation of a $32 \times 32$ Butler matrix in Keysight's Advance Design System (ADS) [11] using micro-strip lines on a Rogers 4350 substrate material with dielectric constant 3.48 and loss tangent 0.004 . For an illustrative frequency of $2.6 \mathrm{GHz}$ and arbitrary $\mathbf{F}_{\mathrm{BB}}$, we have observed that the dynamic signal-dependent loss is approximately zero and that the static loss approaches $2.8 \mathrm{~dB}$.

\section{ENERGY EFFICIENCY}

While reducing the number of RF transceivers, hybrid precoding schemes simultaneously incur in additional power losses as detailed in Section II. A relevant reason for reducing the number of active RF chains is enhancing the transmission's energy efficiency (EE), which is defined as [12]

$$
\epsilon=\frac{R_{\text {sum }}}{P_{\text {tot }}}=\frac{B \sum_{k=1}^{K} \mathbb{E}\left[\log _{2}\left(1+\gamma_{k}\right)\right]}{P_{\mathrm{PA}}+N_{\mathrm{RF}} P_{\mathrm{RF}}+P_{\mathrm{syn}}} \text { bits/Joule, }
$$

where $P_{\text {tot }}$ expressed in Watts (W) refers to the total power employed for transmission and $P_{\mathrm{PA}}=P_{\text {out }} / \eta$ denotes the power consumed by a power amplifier with efficiency $\eta=0.39$ to produce a signal output power of $P_{\text {out }}=40$ $\mathrm{W}$ [12]. Moreover, $P_{\mathrm{RF}}=1 \mathrm{~W}$ and $P_{\text {syn }}=2 \mathrm{~W}$ denote the power consumed by each RF chain and the frequency synthesizer respectively [12]. Note that the effective SINR $\left(\gamma_{k}\right.$ in (10)) of realistic hybrid precoding depends on the specific hardware implementation and will be reduced when compared with their fully digital counterparts due to the dynamic and static losses introduced in Section II. Specifically, (2) can be re-expressed as

$$
\gamma_{k}^{\{\mathrm{FC}, \mathrm{DFT}\}}=\frac{G_{\mathrm{T}}^{\{\mathrm{FC}, \mathrm{DFT}\}}\left|\mathbf{h}_{k}^{\mathrm{H}} \mathbf{f}_{k}^{\{\mathrm{FC}, \mathrm{DFT}\}}\right|^{2}}{G_{\mathrm{T}}^{\{\mathrm{FC}, \mathrm{DFT}\}} \sum_{i \neq k}\left|\mathbf{h}_{k}^{\mathrm{H}} \mathbf{f}_{i}^{\{\mathrm{FC}, \mathrm{DFT}\}}\right|^{2}+\sigma^{2}},
$$

where $\mathbf{f}_{k}^{\{\mathrm{FC}, \mathrm{DFT}\}}$ refers to the $k$-th column of $\mathbf{F}^{\{\mathrm{FC}, \mathrm{DFT}\}}=$ $G_{\mathrm{T}}^{\{\mathrm{FC}, \mathrm{DFT}\}} \mathbf{F}_{\mathrm{BB}} \mathbf{F}_{\mathrm{RF}}^{\{\mathrm{FC}, \mathrm{DFT}\}}$. Here, $G_{\mathrm{T}}^{\{\mathrm{FC}, \mathrm{DFT}\}}$ and $\mathbf{F}_{\mathrm{RF}}^{\{\mathrm{FC}, \mathrm{DFT}\}}$ are given in (8) and (9) for the FC- and DFT-ABFN, respectively.

The power losses of the specific hardware components considered in this letter are shown in Table I. In the following

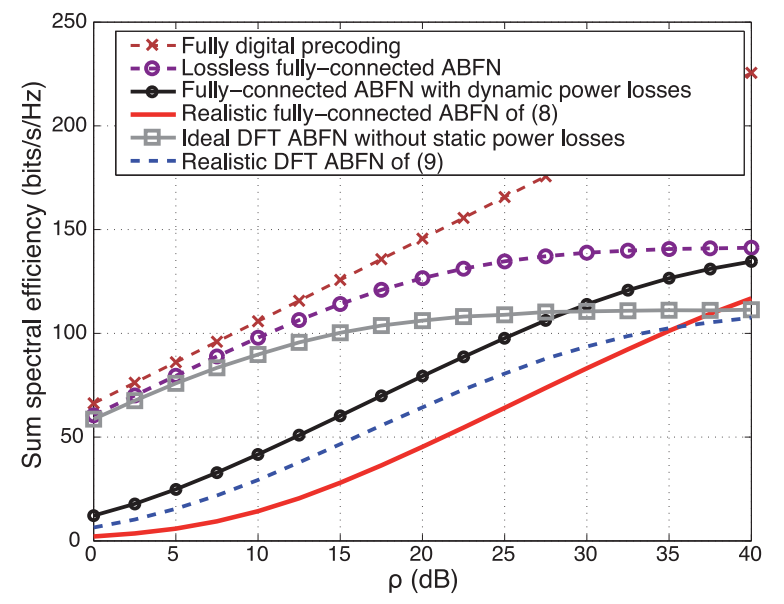

Fig. 3. Sum spectral efficiency (bits/s/Hz) vs. $\rho . N=64, K=12$ with $K_{\{1,2,3\}}=4, N_{\mathrm{RF}}=32$ with $b_{\{1,3\}}=10$ and $b_{2}=12$.

we consider for simplicity that the $(T+1)$-port power combiners and dividers required in large FC-ABFN are built by concatenating $\left\lceil\log _{2}(T)\right\rceil$ three-port structures [7]. Therefore, the overall static losses for the splitting and combining stages of (5) and (7) are given by $L_{\mathrm{D}, \mathrm{dB}}=\bar{L}_{\mathrm{D}}\left\lceil\log _{2}(N)\right\rceil$ and $L_{\mathrm{C}, \mathrm{dB}}=\bar{L}_{\mathrm{C}}\left\lceil\log _{2}\left(N_{\mathrm{RF}}\right)\right\rceil$ respectively, where both $\bar{L}_{\mathrm{S}}$ and $\bar{L}_{\mathrm{C}}$ are provided in Table I.

\section{NUMERICAL RESUltS}

In this section we characterize the performance and EE of realistic ABFN. Although the conclusions derived in the following can be applicable to a vast number of hybrid precoding designs, in the following we focus on the joint spatial division and multiplexing (JSDM) of [4], since it admits both fullyconnected and DFT-based designs. We assume a ULA and adopt the one-ring channel correlation model for microwave frequencies, where the $i, j$-th entry of $\mathbf{R}_{k}$ is given by [4]

$$
\left\{\mathbf{R}_{k}\right\}_{i, j}=\frac{1}{2 \Delta} \int_{-\Delta}^{\Delta} e^{j \frac{2 \pi}{\lambda} d(i-j) \cos (\vartheta+\theta)} d \vartheta,
$$

where $d, \Delta$ and $\lambda$ denote the inter-antenna spacing, angular spread and the wavelength respectively. Moreover, $\theta$ represents the central azimuth angle between the BS and the users. Similarly to [4], we let $d=0.5 \lambda, \Delta=15^{\circ}$ and consider three user groups comprised of $K_{g}=4, g \in\{1,2,3\}$ singleantenna users each with $\theta_{1}=-45^{\circ}, \theta_{2}=0^{\circ}$ and $\theta_{3}=45^{\circ}$. We implement zero-forcing (ZF) precoding with perfect CSI in the digital domain and define $b_{g}$ as the number of RF chains dedicated to serve the users in group $g$, which depends on the eigenvalues of $\mathbf{R}_{k}$ [4]. Without loss of generality, we consider asymmetric power ratios at the output of the power dividers and a sub $5 \mathrm{GHz}$ transmission in this section, since the general conclusions and observations derived in this work are independent of the operating frequency.

Fig. 3 considers $N=64$ and shows the sum spectral efficiency (SE) against increasing $\rho$ for a fully digital precoding system and a hybrid JSDM system implemented via both DFT and FC-ABFN $\left(N_{\mathrm{RF}}=32\right)$. The results depicted in Fig. 3 characterize the performance loss experienced by the realistic FC-ABFN even when ideal analog hardware components are considered, which can be explained by the dynamic power losses introduced by the signal combiners. The performance degradation becomes even more pronounced when the static IL are considered, making a realistic DFT network outperform 


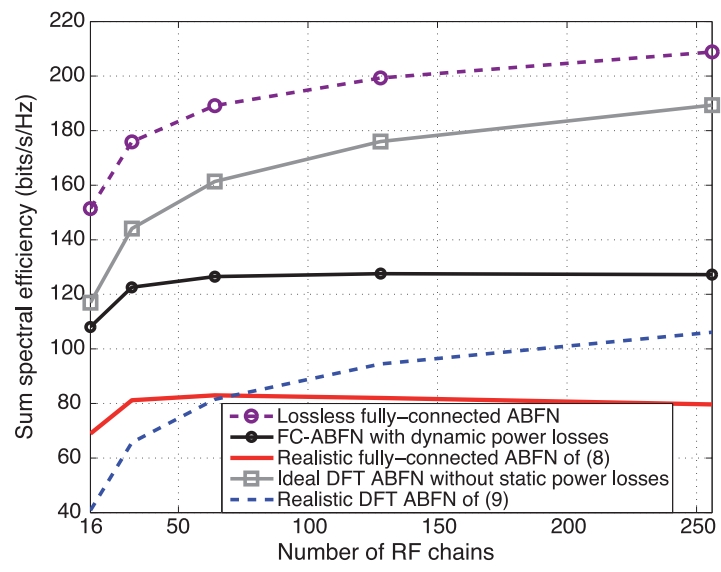

Fig. 4. Sum spectral efficiency (bits/s/Hz) vs. $N_{\mathrm{RF}} \cdot N=512, K=12$ with $K_{\{1,2,3\}}=4$ and $\rho=30 \mathrm{~dB}$.

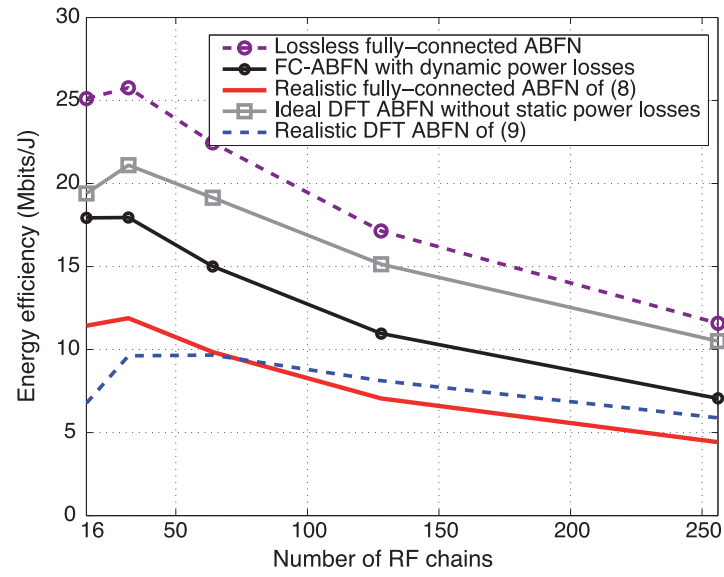

Fig. 5. Energy efficiency (Mbits/Joule) vs. $N_{\mathrm{RF}} \cdot N=512, K=12$ with $K_{\{1,2,3\}}=4$ and $\rho=30 \mathrm{~dB} . B=20 \mathrm{MHz}$ and $P_{\text {out }}=46 \mathrm{dBm}$.

the FC-ABFN for a large range of $\rho$ thanks to its reduced hardware losses. In this context, the results of Fig. 3 also allow concluding that hybrid coupler based DFT ABFN designs will be more spectrally efficient than FC-ABFN for large $N_{\mathrm{RF}}$. This is because, as detailed in Section II-A, the latter architecture introduces power losses that scale proportionally to $N_{\mathrm{RF}}$ even when ideal analog hardware components are considered.

The above observation can be explicitly seen in Fig. 4, which represents the evolution of the sum SE against $N_{\mathrm{RF}}$ for a system with $N=512$ and $\rho=30 \mathrm{~dB}$. The results of this figure explicitly illustrate that the attainable performance of realistic FC-ABFN saturates for large $N_{\mathrm{RF}}$ due to the dynamic power losses in the power combining stage, which linearly grow with $N_{\mathrm{RF}}$ as per (7). Instead, Butler matrix inspired designs enhance the sum SE for increasing $N_{\mathrm{RF}}$ and eventually outperform FC$\mathrm{ABFN}$, hence becoming an attractive alternative for systems requiring large spectral efficiencies, i.e., systems with large $N_{\mathrm{RF}}$. Overall, Fig. 4 involves that a sensible selection of $N_{\mathrm{RF}}$ should consider the power losses' impact on the performance.

Fig. 5 shows the EE against $N_{\mathrm{RF}}$ for the systems considered in Fig. 4. The EE trends allow characterizing the fundamental trade-off offered by hybrid schemes: while a large $N_{\mathrm{RF}}$ generally facilitates an increased design flexibility [3], the overall power consumption is simultaneously increased due to the additional RF hardware components required. Altogether, it can be observed that realistic hybrid schemes with reduced $N_{\mathrm{RF}}$ are capable of offering EE gains over those with large
$N_{\mathrm{RF}}$. This is due to their reduced power losses and the limited performance benefits that can be gained when $N_{\mathrm{RF}}$ grows, as shown in Fig. 4. The results of Fig. 5 also demonstrate that the realistic Butler matrix based implementation constitutes the most energy efficient solution for systems with large $N_{\mathrm{RF}}$.

\section{CONCLUSION}

In this letter we have studied the realistic modeling of ABFN in hybrid precoding systems by accounting for practical power losses on the data rates and the EE. The results shown in this letter demonstrate that the performance of realistic hybrid schemes a) is highly dependent on their hardware implementation, where there is a clear distinction in the performance between Butler-based and fullyconnected designs, and b) is significantly diminished when realistic losses are considered. The consideration of more complex channel models such as those employed at mmWaves constitutes the subject of future work.

\section{ACKNOWLEDGMENT}

The authors would like to thank Dr. Paolo Baracca and Prof. Izzat Darwazeh for their valuable comments on the paper.

\section{REFERENCES}

[1] F. Rusek et al., "Scaling up MIMO: Opportunities and challenges with very large arrays," IEEE Signal Process. Mag., vol. 30, no. 1, pp. 40-60, Jan. 2013.

[2] W. Roh et al., "Millimeter-wave beamforming as an enabling technology for $5 \mathrm{G}$ cellular communications: Theoretical feasibility and prototype results," IEEE Commun. Mag., vol. 52, no. 2, pp. 106-113, Feb. 2014.

[3] O. El Ayach, S. Rajagopal, S. Abu-Surra, Z. Pi, and R. W. Heath, "Spatially sparse precoding in millimeter wave MIMO systems," IEEE Trans. Wireless Commun., vol. 13, no. 3, pp. 1499-1513, Mar. 2014.

[4] A. Adhikary, J. Nam, J.-Y. Ahn, and G. Caire, "Joint spatial division and multiplexing-The large-scale array regime," IEEE Trans. Inf. Theory, vol. 59, no. 10, pp. 6441-6463, Oct. 2013.

[5] P. Sudarshan, N. B. Mehta, A. F. Molisch, and J. Zhang, "Antenna selection with RF pre-processing: Robustness to RF and selection nonidealities," in Proc. IEEE Radio Wireless Conf., Atlanta, GA, USA, Sep. 2004, pp. 391-394.

[6] V. Venkateswaran, F. Pivit, and L. Guan, "Hybrid RF and digital beamformer for cellular networks: Algorithms, microwave architectures, and measurements," IEEE Trans. Microw. Theory Techn., vol. 64, no. 7, pp. 2226-2243, Jul. 2016.

[7] D. M. Pozar, Microwave Engineering. Hoboken, NJ, USA: Wiley, 2011.

[8] S. Wagner, R. Couillet, M. Debbah, and D. T. M. Slock, "Large system analysis of linear precoding in correlated MISO broadcast channels under limited feedback," IEEE Trans. Inf. Theory, vol. 58, no. 7, pp. 4509-4537, Jul. 2012.

[9] H. Hausman, "Understanding mismatch effects in power combining circuits," L-3 Narda-MITEQ, Hauppauge, NY, USA, Tech. Rep., 2006. [Online]. Available: https://miteq.com/docs/ MITEQ_mwrf_mismatch_article.pdf

[10] T. MacNamara, "Simplified design procedures for Butler matrices incorporating $90^{\circ}$ hybrids or $180^{\circ}$ hybrids," IEE Proc. H Microw. Antennas Propag., vol. 134, no. 1, pp. 50-54, Feb. 1987.

[11] Keysight Technologies. Advance Design System. Accessed on Aug. 11, 2016. [Online]. Available: http://www.keysight.com/ en/pc-1297113/advanced-design-system-ads

[12] E. Björnson, L. Sanguinetti, J. Hoydis, and M. Debbah, "Optimal design of energy-efficient multi-user MIMO systems: Is massive MIMO the answer?" IEEE Trans. Wireless Commun., vol. 14, no. 6, pp. 3059-3075, Jun. 2015.

[13] Quinstar Technology Inc. Power Divider-QSP Series. Accessed on Aug. 11, 2016. [Online]. Available: http://quinstar.com/

[14] Anaren Corporation. Power Divider-PD2328J5050S2HF and Hybrid Coupler-X3C22E1-03S. Accessed on Aug. 11, 2016. [Online]. Available: https://www.anaren.com/

[15] Millitech Inc. Hybrid Directional Coupler-CSS-28 and Phase Shifter-VPS-28. Accessed on Aug. 11, 2016. [Online]. Available: http://www.millitech.com/

[16] Analog Devices. Phase Shifter-HMC928LP5E. Accessed on Aug. 11, 2016. [Online]. Available: http://www.analog.com/ 